\section{O tratamento cartográfico da informação em saúde do trabalhador}

\section{Mapping of information on worker's health}

\section{Raul Borges Guimarães'}

Helena Ribeiro"

'Departamento de Geografia da FCT da UNESP

"Departamento de Saúde Ambiental da FSP da USP

Correspondência: Raul Borges Guimarães . Departamento de Geografia da FCT da UNESP - Campus da FCT. Rua Roberto Simonsen, 305 - Presidente Prudente, SP CEP 19060-900. E-mail: raul@fct.unesp.br

\section{Resumo}

Apesar do reconhecimento da importância dos conhecimentos geográficos e do uso das ferramentas de análise espacial nos estudos da saúde coletiva, esse é um campo ainda pouco explorado pelos pesquisadores brasileiros. Em levantamento realizado nas principais revistas científicas que veiculam os resultados de pesquisa em saúde do trabalhador, verificou-se o grande predomínio do uso de tabelas e gráficos como meio de organizar e apresentar os resultados obtidos, e o número reduzido de mapas. Para isso foram examinados todos os artigos publicados em quatro periódicos (Revista de Saúde Pública, Cadernos de Saúde Pública, Revista Saúde e Sociedade e Revista Brasileira de Epidemiologia) no período de 1967 a 2009. Uma vez analisado o conjunto de artigos selecionados no estudo, aqueles que utilizaram representações cartográficas receberam atenção especial. Verificou-se que, embora ainda pouco utilizadas, as ferramentas do geoprocessamento e da geoestatística com suporte em SIG abrem um campo de novas possibilidades no uso da cartografia temática em saúde do trabalhador no Brasil. Contudo, recomenda-se para os editores das revistas científicas o detalhamento de normas técnicas para publicação de figuras cartográficas, assim como a elaboração de pareceres específicos que possam auxiliar os autores em vista das modificações necessárias para a melhoria da qualidade da comunicação visual de mapas e da correlação espacial por meio do tratamento cartográfico.

Palavras-chave: Saúde do trabalhador, Cartografia temática. Informação geográfica. Literatura de revisão. 


\section{Abstract}

Geographic studies and spatial analyses have been recognized in Brazilian public health papers. It is still, however, very little explored by researchers. In a survey of the leading scientific journals covering issues related to Brazilian worker's health, we found the predominant use of charts and tables as a way to organize and present results with a small number of maps. This survey was conducted by examining all papers published in four journals, covering the period from 1967 to 2009 (Revista de Saúde Pública, Cadernos de Saúde Pública, Revista Saúde e Sociedade, and Revista Brasileira de Epidemiologia). After analyzing the set of papers selected for the study, the papers that used maps were given special attention. The tools of geoprocessing and geostatistics with GIS support, although little used, open new possibilities to use thematic cartography in the field of workers' health. However, it is recommended that editors of scientific journals have detailed technical standards as well as specific reports for the publication of cartographic figures aimed at facilitating the modifications necessary for the improvement of the visual quality of maps and of the spatial correlations through cartography.

Keywords: Worker's health. Thematic cartography. Geographic information. Literature review.

\section{Introdução}

A motivação do presente artigo é a discussão do modo como a informação nas pesquisas em saúde do trabalhador tem sido tratada cartograficamente no Brasil. Em levantamento realizado nas principais revistas científicas que veiculam os resultados de pesquisa do tema, verificou-se o grande predomínio do uso de tabelas e gráficos como meio de organizar e apresentar os resultados obtidos, e o número reduzido de mapas. A principal questão que norteia o texto é a relevância das figuras cartográficas não apenas como estratégia de exposição dos resultados, mas no desenvolvimento do raciocínio e da linha argumentativa das pesquisas, facilitando o estudo de correlações espaciais entre condições sociais, ambientais e a saúde do trabalhador.

A representação e visualização do mundo por meio de mapas têm sido objeto de reflexão de vários pesquisadores. Bertin ${ }^{1}$ inventariou os recursos cartográficos, propondo uma semiologia gráfica com base nas categorias de tamanho, forma, valor, textura e orientação. MacEachren ${ }^{2}$ foi além de examinar os mapas apenas como veículo de comunicação visual, estudando o papel da representação cartográfica na forma de estruturar o pensamento acerca das relações espaciais. Por sua vez, o grupo de pesquisa sob a coordenação de Brunet $^{3}$ considerou o mapa tanto como resultado do processo de representação das estruturas elementares da realidade, quanto como ferramenta do processo cognitivo de generalização. Para ele, os modelos gráficos não são meras abstrações, mas resultado de procedimentos racionais de apreensão de relações existentes entre os elementos componentes do mundo sensível.

No campo da saúde coletiva, a cartografia sempre foi um recurso para a formulação de hipóteses etiológicas, conforme Barret $^{4}$. Na primeira metade do século XX, tornaram-se populares vários Atlas mundiais de saúde que retratavam a situação epidemiológica das regiões do globo num contexto de aceleração dos processos de 
transmissão e disseminação de doenças. Essa tendência ganhou tratamento estatístico mais acurado a partir do início da década de 1960, permitindo comparabilidade entre diferentes unidades espaciais, de acordo com Nardocci et al. ${ }^{5}$

Se o uso de mapas nos estudos epidemiológicos tornou-se cada vez mais frequente, a reflexão sobre a qualidade desse recurso transformou-se numa necessidade acadêmica. Desse modo, Gatrell ${ }^{6}$ dedicou um capítulo do seu livro para a discussão dos modelos de mapeamento em geografia da saúde. Além da visualização da distribuição espacial dos dados, ele destaca a importância da geoestatística e da análise espacial, com suporte em sistemas de informação geográfica, para o estudo de alguns temas relevantes da saúde ambiental e saúde dos trabalhadores, como a relação entre a contaminação do solo, a poluição do ar e da água e determinados agravos à saúde (neoplasias, patologias cardiovasculares, entre outros).

$\mathrm{Koch}^{7}$, por sua vez, analisou o mapeamento da distribuição espacial de doenças nos últimos trezentos anos. Sua análise demonstra claramente como o desenvolvimento da epidemiologia não teria sido possível sem a produção cartográfica, uma vez que ela possibilita uma compreensão global das relações entre saúde e ambiente.

No Brasil, Ribeiro ${ }^{8}$ fez referência ao importante trabalho de mapeamento de doenças associadas ao quadro nutricional por Josué de Castro na década de 1940 e das patologias infecciosas e parasitárias por Carlos Lacaz na década de 1970. Segundo Ribeiro, o mapeamento de coeficientes intra-urbanos ganhou atenção mais recentemente ${ }^{9}$.

Por sua vez, Forattini ${ }^{10}$ foi o pesquisador brasileiro que desenvolveu uma primeira reflexão metodológica do mapeamento nas pesquisas em saúde pública do país. Considerando que dificilmente o mapeamento poderia ser substituído na análise e formulação de hipóteses sobre o traçado de quadros nosológicos locais e regionais, ele dedicou especial atenção às formas de representação da distribuição espacial dos fenômenos de interesse epidemiológico, destacando dois grupos principais de mapas: os pontilhados ou dot maps, e os de coeficientes ou rate maps. No primeiro caso, o autor destacou a possibilidade de identificação de conglomerados em áreas, apesar das limitações para a análise dos dados para o conjunto da população. Para essa finalidade, ele recomendou o segundo tipo de mapa, possibilitando o estudo das frequências em diferentes áreas. Preocupado com as variações espaciais das doenças, Forattini também reagrupou os mapas em estáticos ou dinâmicos e discutiu a relevância dos mapas para a comparação entre diferentes áreas e, principalmente, as formas de representação das variações espaciais das doenças no decorrer do tempo.

Atualmente, Barcellos e sua equipe são referências importantes no assunto. A produção desses autores reforça a relevância da análise espacial para a melhor compreensão do processo saúde-doença. Para isso eles chamam a atenção para a escolha correta da escala em função da questão ambiental a ser analisada ${ }^{11}$ e para a necessidade do tratamento das bases para o uso adequado das ferramentas do geoprocessamento na análise espacial ${ }^{12}$.

Apesar do reconhecimento da importância do uso das ferramentas de análise espacial nos estudos da saúde coletiva, esse é um campo ainda pouco explorado pelos pesquisadores brasileiros. $\mathrm{Na}$ análise dos 1.174 posters apresentados no IV Congresso Brasileiro de Epidemiologia realizado no Rio de Janeiro em julho de 1998, Rojas et al. ${ }^{13}$ já haviam ficado surpresos com a escassez de mapas nas áreas de saúde ambiental e saúde ocupacional. Esses autores constataram que, quando utilizados em posters destas áreas, os mapas eram quase meramente ilustrativos, incluindo figuras com a localização do Estado ou município em que se desenvolveu o trabalho, ou a localização de elementos espaciais da amostragem. Nos últimos 10 anos, observou-se a incorporação crescente de técnicas e procedimentos de correlação espacial na área de saúde, particularmente em estudos ecológicos, segundo 
Carvalho e Souza-Santos ${ }^{14}$. As figuras cartográficas utilizadas em artigos sobre a saúde do trabalhador nos periódicos acadêmicos brasileiros não são muito distantes desta tendência, como veremos a seguir.

\section{Procedimentos metodológicos}

O presente estudo teve como objetivo analisar o uso do tratamento cartográfico da informação em artigos científicos da área da saúde do trabalhador no Brasil. Para isso foram examinados quatro periódicos: Revista de Saúde Pública (RSP), editada pela Faculdade de Saúde Pública da Universidade de São Paulo e a mais antiga da área (1967); Cadernos de Saúde Pública (CSP), editado desde 1985 pela Escola Nacional de Saúde Pública da Fundação Oswaldo Cruz; Revista Saúde e Sociedade, editada desde 1992 pela Faculdade de Saúde Pública da Universidade de São Paulo e Associação Paulista de Saúde Pública; e Revista Brasileira de Epidemiologia (RBE), criada em 1998 pela Associação Brasileira de Pós-Graduação em Saúde Coletiva (Abrasco).

Foram examinados todos os artigos desses periódicos, disponíveis na SciELO - Scientific Eletronic Library Online, ${ }^{* * *}$ biblioteca virtual para acesso a periódicos científicos na Internet publicados no período de 1967 a 2009. Os termos "saúde do trabalhador", "saúde no trabalho", "mapa de risco", "risco ocupacional", "fator de risco", "análise espacial", "distribuição espacial" e "mapeamento" foram levantados em todos os campos de busca (título, resumo e palavras-chave do artigo). A análise também incluiu a quantificação de títulos por ano, uma análise qualitativa dos temas e seleção de artigos com algum tratamento cartográfico das informações.

Uma vez analisado o conjunto de artigos selecionados no estudo, aqueles que utilizaram representações cartográficas receberam atenção especial. Em função das características dos fenômenos espaciais analisados e da escala cartográfica adotada foram verificados os tipos de objeto geográfico representados nos mapas. Além da análise da adequação do modo de implantação dos objetos geográficos levou-se em consideração os elementos da linguagem visual empregados, tais como a forma e o tamanho dos objetos.

Por fim, os mapas dos artigos selecionados foram analisados a partir dos níveis de leitura cartográfica propostos por Libault ${ }^{15}$, expressos no Quadro 1. Segundo este autor, cabe perguntar qual é o propósito do tratamento cartográfico da informação. Em um nível elementar, a preocupação central do "mapeador" é apenas de localização. Quando a preocupação envolve a correlação dos dados, o mapeamento é considerado de segundo nível ou de análise espacial. Por fim, se a representação cartográfica possibilita a construção de tipologias e o tratamento gráfico é um suporte para o julgamento e avaliação do pesquisador, obtém-se um nível mais elevado de leitura: a síntese.

\section{Resultados}

O levantamento realizado nas revistas acadêmicas resultou no conjunto de 64 artigos, distribuídos entre as revistas conforme o Gráfico 1. Dentre os periódicos analisados,

Quadro 1 - Níveis de Leitura Cartográfica

Chart 1 - Cartographic reading levels

\begin{tabular}{|l|l|}
\hline Nível & Habilidades cognitivas \\
\hline 1 - elementar & $\begin{array}{l}\text { Identifica e localiza } \\
\text { (o quê? onde?) }\end{array}$ \\
\hline 2 - de correlação & $\begin{array}{l}\text { Estabelece relações entre 2 ou mais elementos } \\
\text { (com quem? por quê?) }\end{array}$ \\
\hline $3-$ de síntese & $\begin{array}{l}\text { Classifica (tipologia) e julga } \\
\text { (como? para quê?) }\end{array}$ \\
\hline
\end{tabular}


a Revista de Saúde Pública é o principal veículo de divulgação das pesquisas realizadas na área da saúde do trabalhador. Do total dos artigos selecionados, 36 (56,3\%) foram publicados nesta revista. Quando ela era o único periódico em circulação dentre aqueles analisados, foram publicados 10 artigos relacionados à saúde ocupacional. Mesmo com o surgimento das outras revistas, a Revista de Saúde Pública concentrou a publicação sobre o tema, reunindo 26 dos artigos analisados no período de 1985 a 2009, seguida de 15 artigos publicados na Revista Brasileira de Epidemiologia, 10 artigos nos Cadernos de Saúde Pública e 3 artigos publicados na revista Saúde e Sociedade. Ainda, de acordo com o gráfico 1, observa-se que, a partir de 2001, ocorreu um aumento significativo de publicações sobre saúde do trabalhador nas quatro revistas, totalizando 45 artigos (70,3\%) do conjunto analisado.

Apenas quatro dos artigos selecionados apresentavam algum tratamento cartográ- fico das informações a respeito da saúde dos trabalhadores. O primeiro deles foi publicado nos Cadernos de Saúde Pública, em 1997 (Facchini et al. ${ }^{16}$ ), o segundo na Revista de Saúde Pública, em 2002 (Leal e Wunch Filho ${ }^{17}$ ), o terceiro neste mesmo periódico, em 2008 (Zangitolani et al. ${ }^{18}$ ), e o quarto artigo na Revista Brasileira de Epidemiologia, em 2009 (Bedor et al. ${ }^{19}$ ). As figuras cartográficas destes quatro artigos foram avaliadas, conforme os níveis de leitura propostos por Libault ${ }^{15}$, e de outros parâmetros importantes da representação temática ${ }^{20,21}$, como o modo de implantação dos objetos geográficos (pontual, linear, zonal), a escala geográfica (local, municipal, regional), as variáveis da comunicação visual (pictórica, forma, valor) e os procedimentos metodológicos (mapeamento participativo, plotagem, elaboração de cartograma, modelagem isarítmica). Todos estes elementos foram utilizados para a organização do Quadro 2, com os níveis de leitura cartográfica dos artigos avaliados.
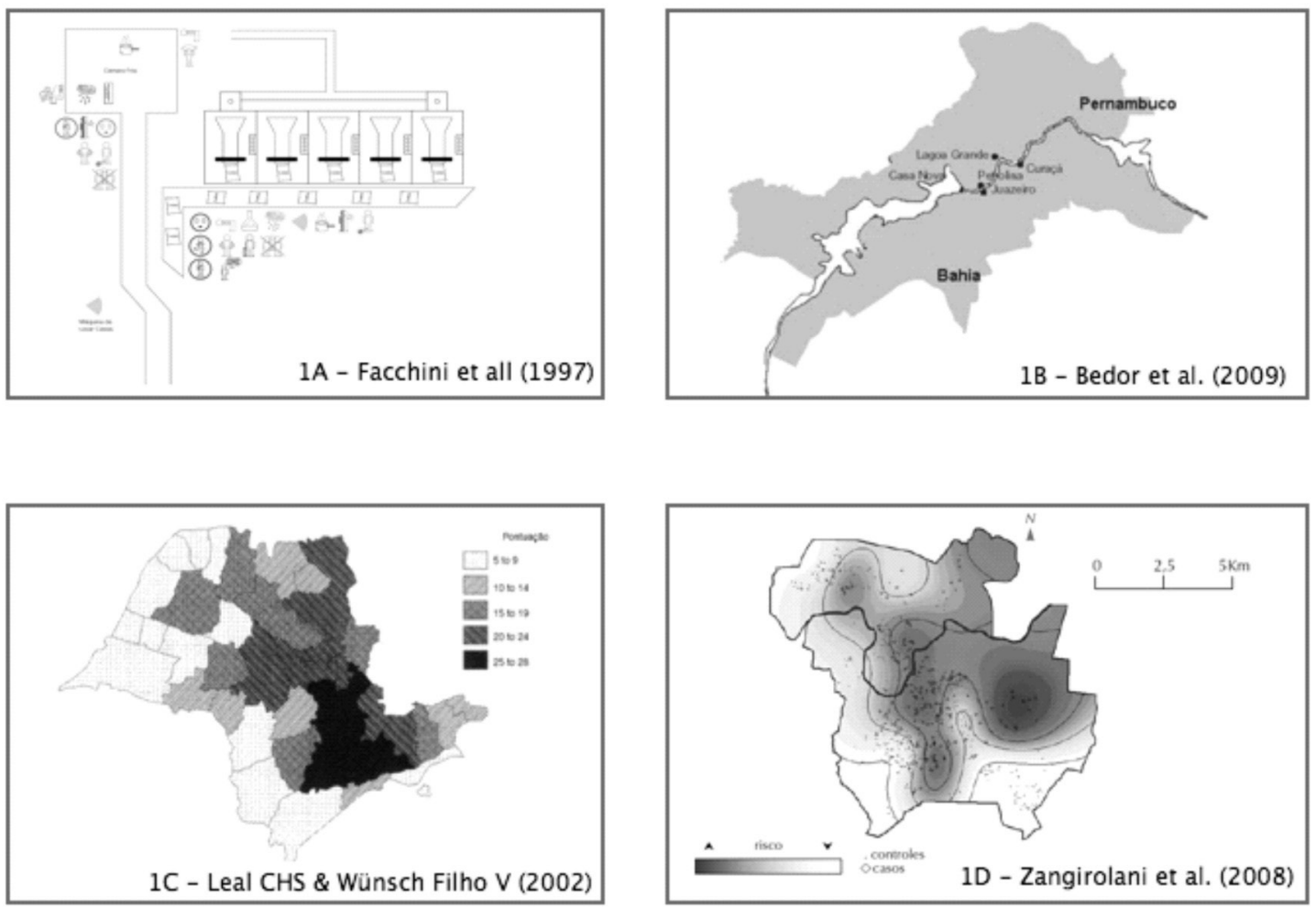

Gráfico 1 - Artigos analisados nas revistas científicas. Graph 1 - Analysed articles in scientific journals. 
Quadro 2 - Níveis de leitura cartográfica - classificação dos artigos pesquisados .

Chart 2 - Cartographic reading levels - classification of papers surveyed.

\begin{tabular}{|c|c|c|c|c|c|}
\hline Artigo & $\begin{array}{c}\text { Nível de leitura } \\
\text { cartográfica }\end{array}$ & $\begin{array}{c}\text { Modo de } \\
\text { implantação }\end{array}$ & Escala & $\begin{array}{c}\text { variáveis da } \\
\text { comunicação } \\
\text { visual }\end{array}$ & $\begin{array}{l}\text { Procedimentos } \\
\text { metodológicos }\end{array}$ \\
\hline $\begin{array}{l}\text { Cad Saúde Públ 1997, } \\
\text { 13(3): 497-502 }\end{array}$ & Elementar & $\begin{array}{l}\text { Pontual } \\
\text { Linear } \\
\text { Zonal }\end{array}$ & local & pictórico & $\begin{array}{l}\text { Mapeamento } \\
\text { participativo }\end{array}$ \\
\hline $\begin{array}{l}\text { Rev Bras Epidemiol 2009, } \\
\text { 12(1): 39-49 }\end{array}$ & Elementar & $\begin{array}{c}\text { Pontual } \\
\text { Linear } \\
\text { Zonal }\end{array}$ & Regional & forma & plotagem \\
\hline $\begin{array}{l}\text { Rev Saúde Pública 2002, } \\
36(4): 400-8\end{array}$ & Correlação & Zonal & Estadual & Valor & $\begin{array}{l}\text { método } \\
\text { coroplético }\end{array}$ \\
\hline $\begin{array}{l}\text { Rev Saúde Pública 2008, } \\
\text { 42(2): 287-93 }\end{array}$ & Síntese & $\begin{array}{l}\text { Pontual } \\
\text { Linear }\end{array}$ & Municipal & Valor & $\begin{array}{l}\text { método } \\
\text { isarítmico }\end{array}$ \\
\hline
\end{tabular}

\section{Discussão}

Apesar do número reduzido de artigos de saúde do trabalhador com tratamento cartográfico da informação $(6,3 \%$ do total de artigos selecionados nas revistas), pôdese identificar uma evolução gradativa do uso destes recursos no estudo da saúde do trabalhador. Isto é evidente com a comparação entre as figuras elaboradas (Figura 1) e o cruzamento dos elementos avaliados (Quadro 2).
O primeiro artigo com tratamento cartográfico ${ }^{16}$ é um exemplo interessante das potencialidades do uso da representação temática em pesquisas da área da saúde do trabalhador. O mapa apresentado é resultado da preocupação com o aprimoramento da análise do risco em ambientes de trabalho. Esses autores discutiram uma proposta de metodologia de mapeamento com a participação dos trabalhadores, gerando um modelo da planta da fábrica, com a representação pictórica dos postos de trabalho

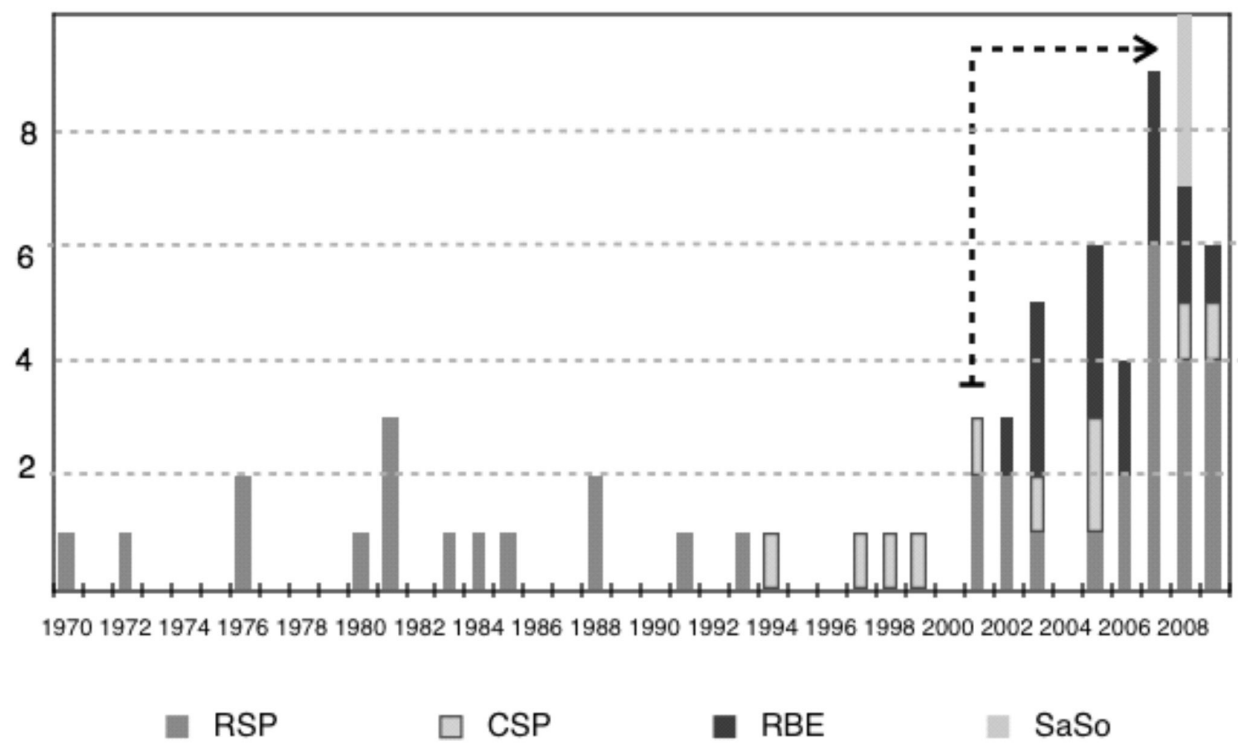

Figura 1 - Artigos analisados: representações cartpgráficas.

Figure 1 - Articles analyzed: cartographic models 
de maior risco (Figura 1A). Trata-se de um produto cartográfico de leitura elementar, uma vez que a representação apenas indica a localização dos objetos por tipo de risco. Mas a simplicidade do mapa não invalida o seu valor para o maior envolvimento dos trabalhadores no diagnóstico e monitoramento dos planos de prevenção dos danos à saúde nos ambientes de trabalho. Pelo contrário, a qualidade do mapa não se mede apenas pelo grau de precisão e de conteúdo técnico, mas também por outros atributos, que devem ser considerados na avaliação da qualidade dos produtos cartográficos, e que foram considerados pelos autores do mapa como a legibilidade ou facilidade de comunicação e a exatidão ou fidelidade às informações levantadas no campo ${ }^{22}$. Afinal, a simplificação é um componente fundamental do método cartográfico que é, acima de tudo, seletivo e generalizado.

Isto não quer dizer que este procedimento, simples e elementar, seja adequado em todas as situações. O nível de precisão e detalhamento de um mapa depende do problema enfocado pela pesquisa, das informações possíveis de serem localizadas e do grau de exatidão aceitável na escala do mapa. Nestes casos, a acurácia do tratamento cartográfico é um atributo imprescindível, como no caso do trabalho de Bedor et al. ${ }^{19}$. Esses autores apresentam no artigo um mapa para localizar os municípios onde foram coletados os dados de intoxicação por agrotóxico no pólo fruticultor do Vale do São Francisco, indicados por pontos nos distritos-sede (Figura 1B). O estudo tinha como objetivo descrever os danos à saúde e ao ambiente decorrente do uso de agrotóxicos. Por meio da aplicação de 300 questionários, amostragem definida para garantir a representatividade de todos os tipos de propriedades, o estudo aponta resultados importantes:

- $69 \%$ dos trabalhadores que manuseiam o agrotóxico são empregados e $31 \%$ pequenos proprietários rurais que trabalham diretamente na terra;

- entre as embalagens vazias de agrotóxicos, $7 \%$ são queimadas, $13 \%$ ficam retidas nas propriedades e o restante é devolvido às lojas ou entregue à Associação do Comércio Agropecuário.

A simples comunicação cartográfica das informações obtidas numa figura, com as divisas administrativas e a localização dos lotes onde foram realizadas as entrevistas, agregaria aspectos importantes na discussão dessa situação. Afinal, era relevante no estudo descritivo reconhecer se haveria alguma diferença entre os municípios da Bahia e de Pernambuco ou entre municípios vizinhos e mais distantes em termos do destino das embalagens vazias de agrotóxicos e de quem é o sujeito que manuseia os produtos, ou seja, os empregados ou pequenos proprietários rurais. Essas eram informações disponíveis nos questionários aplicados e que poderiam receber algum tratamento cartográfico, o que permitiria maior aprofundamento da análise dos dados coletados no campo.

Contudo, o mapa carece de uma delimitação mais precisa dos limites entre os municípios e da divisão interestadual entre Pernambuco e Bahia, na medida em que a área de estudo teve como recorte físicoterritorial os lotes de produtores do curso médio da bacia hidrográfica. Neste sentido, a figura quase se presta apenas à ilustração $\mathrm{e}$ não dá suporte para a avaliação do componente espacial do problema analisado. É aí que a disseminação do geoprocessamento e a de aplicativos computacionais de cartografia digital tem se mostrado como uma grande alternativa para facilitar o uso do método cartográfico pelos pesquisadores da saúde do trabalhador. Seja pela diminuição dos custos, pelo desenvolvimento de plataformas mais amigáveis ou mesmo pela disponibilização gratuita de bases cartográficas digitais pelo IBGE e pelo INPE, as condições serão cada vez mais favoráveis para o melhor aproveitamento dos recursos da cartografia no tratamento das informações das pesquisas.

Isto já pôde ser observado nos mapas produzidos por Leal e Wunch Filho ${ }^{17}$, que fizeram uso do programa computacional 
do EpiMap (programa computacional do Center for Disease Control and Prevention, dos EUA), e por Zangirolani et al. ${ }^{18}$, que utilizaram ferramentas de geoestatística do ArcView (programa computacional do instituto americano ESRI).

No primeiro caso (Figura 1C), os autores elaboraram um tipo de rate map com coeficientes padronizados de mortalidade de leucemia, procurando estabelecer relações com os níveis de industrialização das regiões administrativas do estado de São Paulo. O recurso cartográfico utilizado pelos autores foi a elaboração de cartogramas pelo método coroplético, uma vez que as áreas das regiões administrativas foram transformadas em superfícies estatísticas ${ }^{23}$. Os autores utilizaram este artifício para explorar possíveis relações ecológicas entre industrialização e doença. Esta forma de tratamento cartográfico da informação foi suficiente para se produzir um mapeamento de segundo nível (correlação) e confirmar o que também foi possível analisar por meio da estatística. Ou seja, o método cartográfico demonstrou ser tão eficiente na análise de correlação quanto métodos mais usuais de tratamento estatístico dos dados.

Por sua vez, os autores demonstraram conhecimento das limitações deste tipo de abordagem cartográfica, uma vez que as divisas entre as regiões representadas pelo método coroplético apresentam descontinuidade de unidade observacional de uma área para a outra, o que pode significar uma divisão artificial da realidade. Para isto, os autores fizeram referência à necessidade de definição de áreas em função dos atributos e da dinâmica do fenômeno estudado.

Esse modo de tratamento cartográfico da informação pode ser observado no trabalho de Zangirolani et al. ${ }^{18}$ (Figura 1D). Trata-se de um estudo geoestatístico com suporte em SIG com o objetivo de analisar a distribuição espacial do risco de acidente do trabalho controlado por variáveis nutricionais e outras co-variáveis. Utilizando como fonte o cadastro de atendimento ambulatorial de trabalhadores acidentados e residentes no município de Piracicaba, no
Estado de São Paulo, em centro de ortopedia e traumatologia especializado, foi realizada análise exploratória dos dados, observandose a distribuição espacial de frequência das variáveis estudadas. A partir destes resultados, calculou-se a posição central e a dispersão das variáveis contínuas, possibilitando a elaboração de um mapa-síntese (Figura 1D) com a aplicação do método isarítmico.

Segundo Martinellii ${ }^{23}$ o método isarítmico resulta da representação da superfície estatística por isolinhas (linhas que unem pontos de igual valor). Assim, valores tomados em pontos dispersos na superfície do mapa possuem uma localização $(\mathrm{X}, \mathrm{Y})$ e uma terceira dimensão (Z). No artigo de Zangirolani et al. ${ }^{18}$ este procedimento foi adotado para estabelecer a intersecção entre os endereços das empresas com acidentados, setorizando o espaço urbano por grau de risco (superfície tridimensional). É por isto que este mapa foi considerado de terceiro nível, uma vez que é resultado de um esforço de síntese, por meio da recuperação da superfície de valores a partir de dados pontuais dispersos.

Se a cartografia digital facilitou o manuseio de programas de suporte SIG e incorporou procedimentos de geoestatística no processamento de dados (Figuras 1C e 1D), ainda é preciso avançar no acabamento das figuras publicadas. Para quem não conhece as regiões administrativas do Estado de São Paulo, ou a cidade paulista de Piracicaba, elementos básicos de comunicação cartográfica facilitariam a correlação espacial complementar à correlação estatística, como a indicação de vias principais e circulação e da área urbana central.

\section{Considerações finais}

Nos artigos analisados, os dados espaciais não foram apenas do tipo quantitativo, mas também qualitativos. Os dados quantitativos são resultados de medidas ou contagens efetuadas acerca dos fenômenos estudados. Neste caso, por exemplo, os dados referem-se à distância entre os objetos geográficos, bem como à concentração ou 
dispersão, às médias, às frequências e às flutuações. Os dados qualitativos referem-se às características dos objetos geográficos, tais como localização e distribuição no espaço, vizinhança e conectividade.

A escolha de qual representação cartográfica é mais adequada depende do poder de síntese do assunto que se quer tratar, ou dos propósitos da pesquisa. Nos quatro artigos analisados, do conjunto de 64 selecionados, os autores utilizaram o tratamento cartográfico da informação para analisar problemas de localização e de relações espaciais, problemas de qualificação e de diferenciação entre áreas, problemas de classificação e comparação entre lugares (grau de risco) e problemas de proporções e valores de superfícies estatísticas. Estes são alguns problemas interessantes que podem ser explorados pelo tratamento cartográfico das informações em pesquisas na área da saúde do trabalhador.

Ainda que os mapas sejam quase inexistentes nos estudos em saúde do trabalhador, o uso do geoprocessamento e de ferramentas de geoestatística, com suporte em SIG, abre um campo de novas investigações. Isto exige maior discussão dos limites $\mathrm{e}$ potencialidades da cartografia, bem como das dificuldades encontradas para tornar as geotecnologias mais amigáveis aos pesquisadores brasileiros. Várias iniciativas nesta direção estão em desenvolvimento, como a formação de redes cooperativas interdisciplinares, envolvendo especialistas de diversas áreas (epidemiologia, geografia, estatística, ciências da computação etc.), como ocorre no projeto Saudável, dentre outros exemplos citados por Carvalho e Souza-Santos ${ }^{18}$.

Contudo, a impressão que fica da análise dos mapas é a de que talvez a cartografia digital tenha reforçado a idéia de que o tratamento gráfico da informação responde a processamentos automáticos pré-estabelecidos, não havendo a necessidade de fazer uso da arte expressiva dos mapas como um recurso de comunicação e cognição. Da mesma forma, talvez o impacto provocado pelas geotecnologias (sensoriamento re- moto, sistemas de informação geográfica e grande oferta de pacotes de geoestatística) tenha reforçado a associação do mapeamento com abordagens quantitativas.

A despeito do desenvolvimento de novas tecnologias, que culminaram inclusive com uma nova definição de Cartografia pela Associação Cartográfica Internacional, com relação ao mapeamento temático, ainda é válida a definição de Joly ${ }^{22}$, segundo a qual cartografia é a arte de conceber, de levantar, de redigir e de divulgar os mapas. Assim, o mapeamento também pode ser um caminho a ser considerado na análise de fenômenos que não sofrem avaliação matemática. Neste caso, o melhor tratamento para se eliminar imprecisões ou facilitar a conversão do subjetivo ao objetivo, é submeter o fenômeno à prova da cartografação ${ }^{15}$. Desde que respeitadas as regras da linguagem visual, que considera as limitações do olho humano diante dos objetos ao seu redor, a correlação cartográfica certamente complementa a correlação estatística, como demonstra o artigo de Leal eWunch Filho ${ }^{17}$.

A representação dos elementos geográficos, por meio de linhas, formas e cores, coloca esse processo de tomada de decisão não apenas no campo do conhecimento científico, mas envolve o domínio da linguagem apropriada aos problemas tratados nas diversas pesquisas e expressos de diferentes maneiras pelas figuras cartográficas dos artigos científicos. Assim, nas aplicações em saúde do trabalhador em questão, é o pesquisador que conhece o assunto e o modelo que vai representar - o risco ocupacional, por exemplo. É ele que deve discernir para acrescentar ou suprimir detalhes, segundo a escala e a finalidade do seu mapa.

Tais conclusões baseiam-se no fato de que os documentos cartográficos não são simplesmente apreendidos de modo intacto do papel para a mente do leitor. Pelo contrário, quando o leitor observa uma figura cartográfica, exerce um papel ativo de decodificação, interpretação e avaliação. É por causa disto que, ao olhar para os mapas, $o$ leitor não mobiliza apenas a sua capacidade de visualização da realidade a partir de sua 
representação gráfica. Eles também exigem capacidade de leitura dos códigos específicos da linguagem cartográfica, potencializando o uso de cartas temáticas na análise de interações entre o espaço produzido, as condições ambientais e o risco à saúde dos trabalhadores.

Pelo exposto acima, recomenda-se aos editores das revistas científicas o detalha- mento de normas técnicas para publicação de figuras cartográficas nas orientações para apresentação de artigos, assim como o encaminhamento a pareceristas especializados em cartografia, que possam auxiliar os autores nas modificações necessárias para a melhoria da qualidade da comunicação visual de mapas e da correlação espacial por meio do tratamento cartográfico.

\section{Referências}

1. Bertin J. Semiology of graphics: diagrams, networks, maps. Madison: University of Wisconsin Press; 1983.

2. MacEachren AM. How maps work - representation, visualization and design. New York/London: Guilford Press; 2004.

3. Brunet R. Le dechiffrement du monde. Paris: Belin; 2001.

4. Barret F. Disease and geography - the history of an idea. Toronto: York University; 2000.

5. Nardocci AC, Rocha AA, Ribeiro H, Assunção JV, Colacioppo S, Paganini WS. Saúde ambiental e ocupacional. In Rocha AA, César CLG (eds.). Saúde Pública-bases conceituais. São Paulo: Atheneu; 2008.

6. Gatrell A. Geographies of health. Oxford: Blackwell; 2002.

7. Koch T. Cartographies of disease - maps, mapping and medicine. Redlands, California: Esri Press; 2005.

8. Ribeiro, H. Geografia para a saúde nas cidades e a questão da escala. In: Anais do $10^{\circ}$ Encontro de Geógrafos da América Latina. São Paulo: USP; 2005. p. 50.

9. Ribeiro H. Mapeamento das causas de morte no município de São Paulo. Bol Paul Geogr 1988; 66: 85-90.

10. Forattini OP. Ecologia, epidemiologia e sociedade. São Paulo: Artes Médicas/Edusp; 1992.

11. Barcellos C, Bastos FI. Geoprocessamento, ambiente e saúde: uma união possível? Cad Saúde Pública 1996; 12(3): 389-97.

12. Skaba DA, Carvalho MS, Barcellos C, Martins PC, Terron SL. Geoprocessamento dos dados da saúde: o tratamento dos endereços. Cad Saúde Pública 2004; 20(6): 1753-6.

13. Rojas LI, Barcellos C, Peiter P. Utilização de mapas no campo da epidemiologia no Brasil: reflexões sobre trabalhos apresentados no IV Congresso Brasileiro de Epidemiologia. Informe Epidemiológico do SUS 1999; 8(2): 27-35.
14. Carvalho MS, Souza-Santos R. Análise de dados espaciais em saúde pública: métodos, problemas, perspectivas. Cad Saúde Pública 2005; 21(2): 361-78.

15. Libault A. Geocartografia. São Paulo: Companhia Editora Nacional; 1975.

16. Facchini LA et al. Ícones para mapas de riscos: uma proposta construída com os trabalhadores. Cad Saúde Pública 1997; 13(3): 497-502.

17. Leal CHS, Wunsch Filho V. Mortalidade por leucemias relacionada à industrialização. Rev Saúde Pública 2002; 36(4): 400-8.

18. Zangirolani LTO et al. Topologia do risco de acidentes do trabalho em Piracicaba, SP. Rev Saúde Pública 2008; 42(2): 287-93.

19. Bedor CNG et al. Vulnerabilidades e situações de riscos relacionados ao uso de agrotóxicos na fruticultura irrigada. Rev Bras Epidemiol 2009; 12(1): 39-49.

20. Oliveira C. Curso de cartografia moderna. Rio de Janeiro: IBGE; 1988.

21. Raisz E. Cartografia geral. Rio de Janeiro: Editora Científica; 1969.

22. Joly F. A cartografia. Campinas (SP): Papirus; 1990.

23. Martinelli M. Curso de cartografia temática. São Paulo: Contexto; 1991.

Recebido em: 17/11/09 Versão final reapresentada em: 28/07/10 Aprovado em: 31/08/10 